Document downloaded from:

http://hdl.handle.net/10251/166759

This paper must be cited as:

Moya, A.; Navarro, E.; Jaén Martínez, FJ.; González, P. (2020). Fuzzy-description logic for supporting the rehabilitation of the elderly. Expert Systems. 37(2):1-16.

https://doi.org/10.1111/exsy.12464

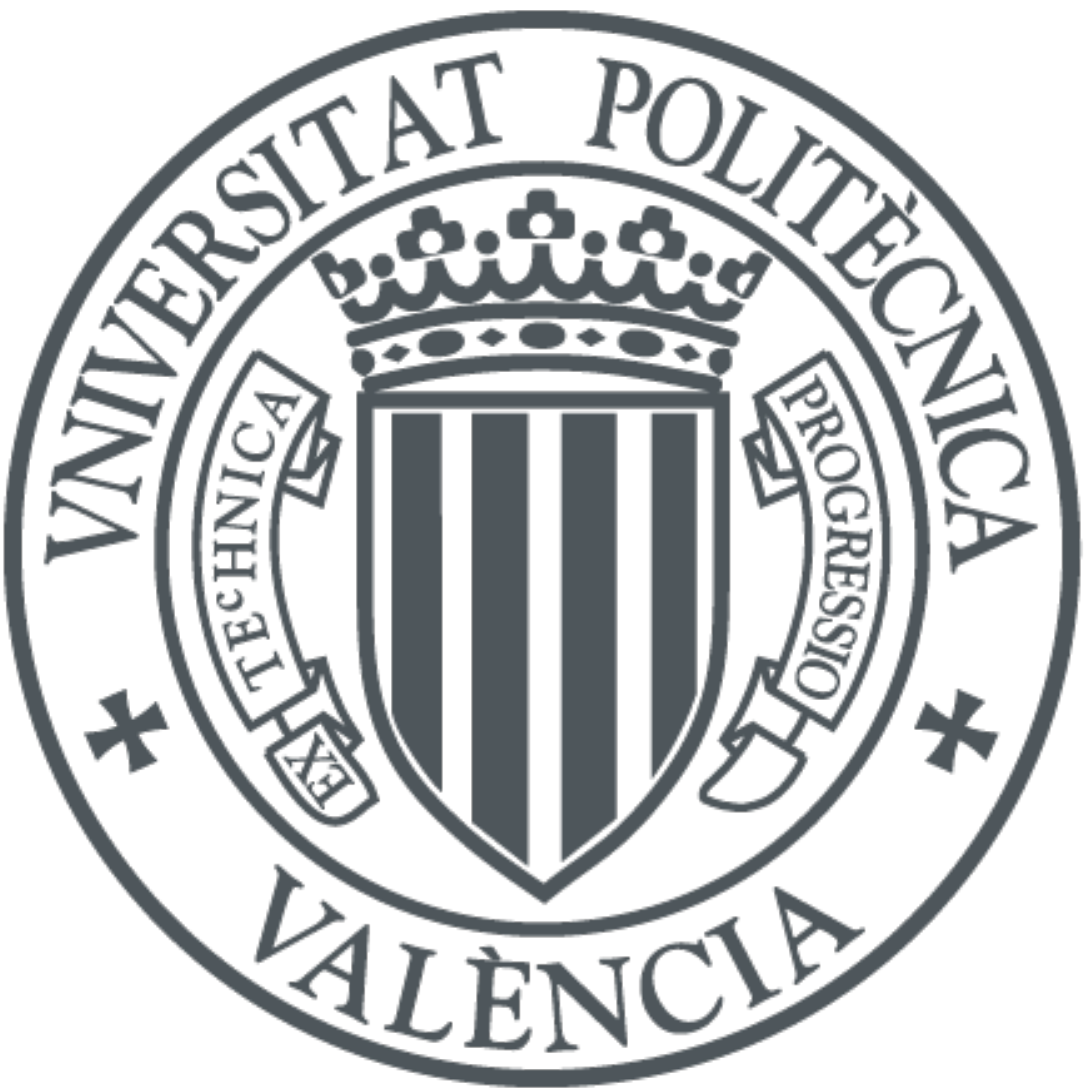

The final publication is available at

https://doi.org/10.1111/exsy.12464

Copyright Blackwell Publishing

Additional Information 


\title{
Fuzzy-Description Logic for supporting the rehabilitation of the elderly
}

\author{
Alejandro Moya \\ LoUISE Research Group, Computing Systems Department, University of Castilla- La Mancha \\ Avda. España s/n, 02071 Albacete (Spain) \\ Alejandro.Moya4@alu.uclm.es \\ Elena Navarro* \\ LoUISE Research Group, Computing Systems Department, University of Castilla- La Mancha \\ Avda. España s/n, 02071 Albacete (Spain) \\ Phone +34 967599200 ext. 2365, fax +34967599343 \\ Elena.Navarro@uclm.es \\ Javier Jaén \\ ISSI, Departamento de Sistemas Informáticos y Computación, Universitat Politécnica de València, \\ Camino de Vera s/n. 46022 Valencia (Spain) \\ fjaen@upv.es \\ Pascual González \\ LoUISE Research Group, Computing Systems Department, University of Castilla- La Mancha \\ Avda. España s/n, 02071 Albacete (Spain) \\ Pascual.Gonzalez@uclm.es
}

\begin{abstract}
According to the latest statistics, the proportion of the elderly $(+65)$ is increasing and is expected to double within the European Union in a period of 50 years. This ageing is due to the improvement of quality of life and advances in medicine in the last decades. Gerontechnology is receiving a great deal of attention as a way of providing the elderly with sustainable products, environments and services combining gerontology and technology. One of the most important aspects to consider by gerontechnology is the mobility/rehabilitation technologies, because there is an important relationship between mobility and the elderly's quality of life. Tele-rehabilitation systems have emerged to allow the elderly to perform their rehabilitation exercises remotely. However, in many cases, the proposed systems neither assist the patients nor the experts about the progress of the rehabilitation. To address this problem, we propose in this paper a fuzzy-semantic system for evaluating patient's physical state during the rehabilitation process based on well-known standard for patients' evaluation. Moreover, a tool called FINE has been developed that facilitates the evaluation be accomplished in a semi-
\end{abstract}

\footnotetext{
${ }^{*}$ Corresponding author
} 
automatic way first asking patients to carry out a set of standard tests and then, inferencing their state by means of a fuzzy-semantic approach using the data captured during the rehabilitation tasks.

Keywords. Fuzzy-Description Logic, elderly, tele-rehabilitation, ontology, gerontechnology.

\section{Introduction}

The world's population is ageing. According to (Nassabi, Akker, \& Vollenbroek-, 2014), the proportion of the elderly $(+65)$ is increasing and is expected to double within the European Union in a period of 50 years. This ageing is due to the improvement of quality of life and advances in medicine in the last decades. According to (Commission, 2006), this social transformation will have implications for nearly all sectors of our society. As stated by the European Commission Information Society and Media "The situation is becoming unsustainable and will only worsen in the future as chronic diseases and demographic change place additional strains on healthcare systems around Europe''. Providing a proper healthcare system is expected from any developed country as this aspect has a great impact on the quality of life of its citizens, especially among the elderly.

One of the services of the healthcare systems is rehabilitation in any of its dimensions such as physical, cognitive or behavioural. The elderly are frequent users of this service as, unfortunately, they are prone to accidents due to their mobility problems, degenerative illness such as Alzheimer, or depression due to isolation. According to (Chiu et al., 2018), traditional rehabilitation treatments are usually administered on a one-to-one basis, which makes them difficult to scale due to the high cost and the dependency on human resources. Besides, traditional rehabilitation treatments required the presence of the elderly in the clinic, causing a waste of money and time for the elderly. As a consequence, the adherence rate to traditional rehabilitation exercises is relatively low. The study carried out by (Shaughnessy, Resnick, \& Macko, 2006) concludes that only $31 \%$ of the prescribed exercises are completed.

According to (Fernández-Caballero, González, \& Navarro, 2017), "Sustainability of an ageing society depends of creating systems that exploit assistive technology and inclusive designs". In this respect, the field of gerontechnology is receiving a great deal of attention being telerehabilitation systems for the elderly one its most rapidly growing areas. These systems allow the elderly to perform their rehabilitation exercises remotely. However, in many cases, the proposed systems neither assist the patients nor the experts about the progress of the rehabilitation. 
To address this problem, we propose in this paper a fuzzy-semantic system (Bobillo, 2008) for evaluating patient's physical state during the rehabilitation process. This evaluation is accomplished in a semi-automatic way first asking patients to carry out a set of standard tests and then, inferencing their state using the data captured during the rehabilitation tasks by means of a fuzzy-semantic approach. This processed information may be used by the specialists to make decisions about the suitability of the proposed rehabilitation process.

The remainder of this paper is structured as follows. Section 2 discusses related works and highlights the novelty of the present work. Section 3 presents our proposal, using a Fuzzysemantic approach for supporting the rehabilitation of the elderly. Section 4 describes the resulting system (FINE - Fuzzy ImpairmeNt Evaluation tool) a fully implemented system that integrates a fuzzy hybrid semantic system for supporting the telerehabilitation process of the elderly. Finally, Section 5 presents our main conclusions and areas of future work.

\section{Related Work}

Gerontechnology is an interdisciplinary field combining gerontology and technology to develop products, environments and services (systems for health, housing, mobility, etc.). According to (Rodríguez, Roda, Montero, González, \& Navarro, 2016), gerontechnology can provide both patients and healthcare organizations with the required solutions. These systems can prevent, delay or compensate those physical and cognitive problems related to ageing and provide new opportunities for the elderly such as leisure, learning, etc. (Bronswijk, Bouma, \& Fozard, 2002) have suggested that all the research, development, and design carried out in the gerontechnology field should be oriented to its end-users from its inception.

According to (Metz, 2000), one of the most important areas to consider is mobility/rehabilitation technologies, because there is an important relationship between mobility and the elderly's quality of life. Reduced mobility is a very limiting factor that prevents our seniors from carrying out everyday activities resulting, in some cases, in important functional impairments. Consequently, gerontechnology in general, and telerehabilitation systems in particular, have experienced a great development in recent years focusing on technologies that analyse the patient's health and adapt the rehabilitation tasks based on the patient's abilities and the expert's performance requirements.

A telerehabilitation or home-based rehabilitation system can be defined as "the application of telecommunication, remote sensing and operation technologies, and computing technologies to assist with the provision of medical rehabilitation services at a distance' (Boucenna et al., 2014). These systems can be really beneficial in the rehabilitation process, according to 
(Karime, Eid, Alja'Am, Saddik, \& Gueaieb, 2014)(Su, Chiang, \& Huang, 2014), because they can eliminate travel distance between the home and the clinic, that can be time consuming and expensive. Besides, they allow for great flexibility because patients can specify their preferences and schedules. According to (Navarro et al., 2018), demand for this type of systems is growing (the ever-increasing costs of looking after an aging population, many of whom suffer chronic diseases) and this fact will cause the development of a more economically-viable homebased treatment program without the presence of an expert, i.e., take rehabilitation beyond hospitals and care centres.

In the literature we may find different proposals focusing on the development of physical telerehabilitation systems. For instance, (Alamri, Cha, \& El Saddik, 2010) propose a framework for Poststroke-Patient rehabilitation called AR-REHAB. It allows users to see real-world scenes and manipulate real objects in their exercises. When the patient has carried out the exercises, the therapist receives feedback allowing him(her) to manually adapt the rehabilitation tasks. This system requires that a therapist is constantly analysing the information offered by the system and adapting manually the tasks because it does not offer any intelligent component that automatically analyses the captured data resulting in a loss of efficiency.

To overcome this problem there are proposals that include some component that turn the system into a smart system. For instance, (Krynicki, Jaen, \& Navarro, 2016) present an Ant colony optimization (ACO)-based recommendation technique to propose activities to people with Acquired Brain Injury, starting with those that have proven to be the most generally successful, and then progressing towards more personalized recommendations, at all times taking into account the user's mental and physical state. They used ACO because it is suitable for establishing paths in a discrete space, so that the search over the space of learning units is not limited to a sequence. but the proposal finds the most suitable unit for a given user when requested. Another example is a rule-based framework, presented by (Zhao, Reinthal, Espy, \& Luo, 2017), that can be used to assess in real-time the quality and quantity of exercises performed by the user. This framework is used to create rehabilitation exercises described as sets of rules about position, angle, etc. of patient's joints. Then the patient has to fulfill the set of rules in order for the exercise to be successful, receiving real-time feedback when any rule is not satisfied.

Despite the benefits of the previous systems, they assume that knowledge about the physical or mental state of a patient is sound and trustable which is not always the case. To overcome the previous problem several telerehabilitation systems have been proposed based on fuzzylogic (Ross, 2004) to adequately represent the uncertainty which arises both from the 
unavoidable lack of precision of data provided by hardware used to capture patients' movements, emotions, etc. and from the estimation process that evaluates the patient actual state based on the rehabilitation tasks he(she) is able to perform.

In this respect, many prior works have proposed telerehabilitation systems that analyse the patient's state, offer adapted automatic therapies and show the progress/improvement of the elderly during the rehabilitation process based on incomplete or uncertain information. In (Karime et al., 2014) an adaptive home-based rehabilitation framework for the rehabilitation of the wrist is proposed. The authors conduct a series of tests to evaluate the effect of tilting the wrist in various angular positions on a number of performance parameters. They propose a fuzzy-logic adaptation engine to support decision making relative to the appropriate intensity level of a rehabilitation task considering the task angle (different tilting positions of the wrist), the velocity and the jerkiness of the wrist. In the work proposed by (Chiu et al., 2018) an Android application is implemented to: (1) collect quantitative data of hand movements to monitor treatment progress; (2) collect statistical data to assess self-rehabilitation progress; (3) motivate the use of an assistive system for hand rehabilitation by using virtual reality and gamebased modalities; and (4) provide automatic feedback on hand rehabilitation progress by means of a computer-aided decision making mechanism. (Zhang, Fang, \& Gu, 2014) present an upperextremity motion evaluation system based on fuzzy logic to provide a reliable solution for objective motion quality assessment. This fuzzy system is based on the automatic Brunnstrom stage classification as well a special device for tracking that authors have developed. Finally, (Su et al., 2014) propose a Kinect-enabled home-based rehabilitation system that use Dynamic Time Warping and fuzzy logic. The main objective of the system is to assist patients while they replicate the movements as were proposed at the medical centre.

According to the previous review it can be concluded that the proposed works focus on the adaptation of the rehabilitation tasks according to the patient's health. Moreover, most of the proposals do not follow standard rehabilitation models when evaluating the patient's physical condition. The evaluation of the patient's health is crucial for the proper functioning of the telerehabilitation systems. In this respect, an accurate analysis of the patient's physical health can provide the therapist with exact data for creating, modifying or eliminating rehabilitation tasks. In order to address the previous shortcomings, this work has been carried out to explore how to support a well-known standard for patients' assessment using fuzzy-description logic (Fuzzy-DL). This will facilitate therapists may adapt the rehabilitation process using accurate information about the patients' state. Moreover, another important aspect considered in the development of the proposal is the guidance of the patients while they carry out the tests. 


\section{Our proposal: Fuzzy-DL for supporting the rehabilitation of the elderly}

As discussed above, our proposal supports the rehabilitation process of the elderly by handling and processing incomplete or uncertain information to give therapists the most accurate information about the actual progress of their patients, so that the most effective therapies may be recommended. In this section, we describe the standards on which we base our approach and the models and inference mechanisms that support the construction of new intelligent rehabilitation recommendation systems.

\subsection{Fugl-Meyer standardization}

The evaluation of the patient cannot be performed in an arbitrary way. This evaluation must be supported by well-defined standards or a therapy expert. In our proposal, we use the FuglMeyer standard (Velozo \& Woodbury, 2011). This standard is focused on physical rehabilitation and it defines 30 tests to evaluate the physical condition of patients and to redirect the rehabilitation tasks. This standard not only proposes a set of tests but also a simple rating scale consisting of three levels: 0 (unable to perform), 1 (partial performance) or 2 (close to normal performance).

In (Hsieh et al., 2007) a reduction of the number of tests is proposed, from 30 down to 12 because of the difficulty to perform all the tests. In Table 1, we can see that the selected tests are grouped by the affected extremity (upper and lower). According to authors, these tests are sufficient to evaluate perfectly the physical condition of the patient.

Table 1. Evaluations selected by (Hsieh et al., 2007).

Upper extremity

Lower extremity

\begin{tabular}{|c|c|}
\hline Shoulder flexion $90^{\circ}$ to $180^{\circ}$ & Heel to opposite knee time \\
\hline Grasp, adduct thumb & Knee (standing) flexion \\
\hline Elbow $90^{\circ}$ pronation/supination & Ankle (sitting) dorsiflexion \\
\hline Elbow $90^{\circ}$ wrist flexion/extension & Knee (sitting) flexion \\
\hline Elbow extension & Ankle plantar flexion \\
\hline Shoulder elevation & Hip flexion \\
\hline \multicolumn{2}{|c|}{$\begin{array}{l}\text { As a first approximation, in our proposal we use the evaluations selected by (Hsieh et al., } \\
\text { 2007), but we enhance the precision of the scoring system. Instead of using a discrete three- } \\
\text { valued ( } 0 \text { - unable to perform, } 1 \text { - partial performance or } 2 \text { - close to normal performance) } \\
\text { scoring system, we use a continuous scoring system in the range [0,1] (0-unable to perform, } 1 \text { - }\end{array}$} \\
\hline
\end{tabular}


normal performance). This way the therapist can accurately distinguish the degree of mobility achieved in a given test in order to monitor the physical condition of the patient more precisely.

\subsection{An ontological description of the rehabilitation}

Ontologies are frequently used whenever a complex and rich knowledge must be described in a computer-based format that may be exploited to verify its consistency or to facilitate reasoning about such knowledge (Antoniou \& Van Harmelen, 2004). To facilitate the development of intelligent rehabilitation systems it is needed an appropriate model to represent, manipulate and access to information about the body and movements of the patient. To achieve this goal, Web Ontology Language 2 (OWL 2) (W3C, 2012) may be used to address issues that include the representation of the semantic, the reasoning, the exchange, the classification, the dependence and the quality of the knowledge about the patient. The ultimate goal pursued is the ability to reason about the patient. For this aim, in the following the ontology that has been created using OWL is presented.

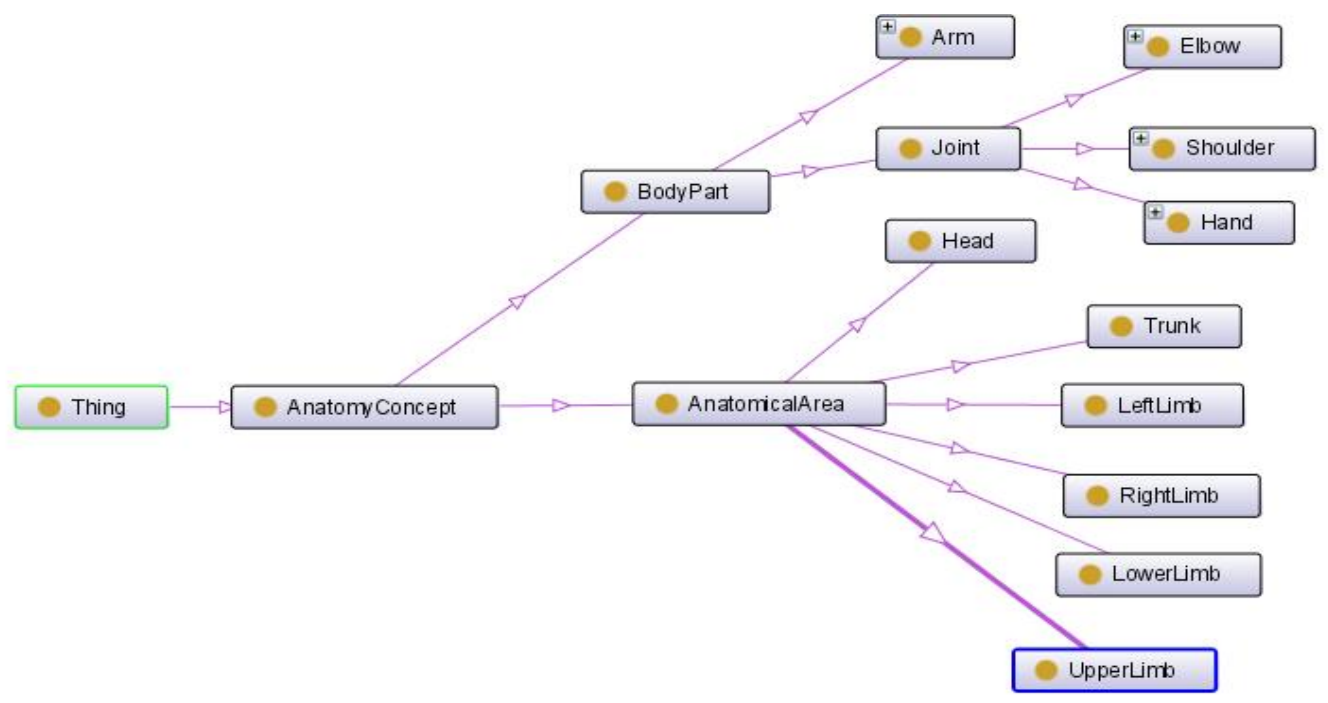

Figure 1. Ontology developed - Hierarchy of entities

An ontology of the human-body has been created using OWL2. As an example, the semantic definition of the upper-limb and its parts is shown in Figure 1. The most generic element is the entity "AnatomyConcept" that defines a general concept from which two entities derive: "AnatomicalArea" and "BodyPart". Anatomical areas are defined to allow the decomposition of a body into subareas, so that we can infer rehabilitation information not only about specific body parts but also about complete body regions. The different areas defined in our semantic model are: "Head", "Trunk", "LeftLimb", "RightLimb", "LowerLimb" and "UpperLimb". The "BodyPart" class is further specialized into concepts such as "Joint" and "Arm" and "Joint" is 
further specialized into concepts such as "Elbow", "Shoulder" and "Hand". This conceptual hierarchical decomposition defines the categories over which the reasoning tasks will take place.

However, semantic reasoning is not only defined in terms of ontological classes but also in terms of the properties that relate them. The relationships defined in our semantic model are listed in Table 2. As can be observed, the relations have been defined taking into account how each anatomical structure is made up of different components. For instance, an Arm is made up by Hand, Elbow and Shoulder and this is specified by the relations hasHand, hasElbow and hasShoulder.

Not only relationships can be defined between entities, but also between an entity and a primitive type. As can be shown in Table 3, this facilitates that it can be specified which flexion, or extension may have, as Joint has different relations to primitive types.

Table 2. Ontology developed - Relations between entities

\begin{tabular}{l|ll} 
Relation & Origin entity & Target entity \\
\hline hasHand & Arm & Hand \\
hasElbow & Arm & Elbow \\
hasShoulder & Arm & Shoulder \\
hasArm & UpperLimb & Arm
\end{tabular}

Table 3. Ontology developed - Relations between entities and values

\begin{tabular}{l|lc}
\multicolumn{1}{l}{ Relation } & Origin entity & Target entity \\
\hline hasFlexion & Joint & *real* \\
hasExtension & Joint & real* $^{*}$ \\
hasAxisY & Joint & $*$ real* \\
hasAverageSpeed & AnatomyConcept & *real*
\end{tabular}

Table 4. Ontology developed - instances created

\begin{tabular}{l|ll} 
Instance & Entity & Probability of existence \\
\hline upper_limb & UpperLimb & 1 \\
right_hand & Hand & 1 \\
right_shoulder & Shoulder & 1 \\
right_arm & Arm & 1 \\
right_elbow & Elbow & 1 \\
left_hand & Hand & 1 \\
left_shoulder & Shoulder & 1 \\
left_arm & Arm & 1 \\
left_elbow & Elbow & 1
\end{tabular}


Finally, once the ontological concepts and relationships have been defined, the model has to be instantiated by defining the instances belonging to each class. In our proposed ontology, we have defined the instances described in Table 4. It can be observed that each instance has a probability of existence of $100 \%$ to describe that a subject has each one of these parts of the body. This may be used also to describe when a subject does not have any of them because of an amputation or a congenital problem, establishing then that the probability of existence is 0 . As can be observed, right_hand, left_hand, right_elbow, left_elbow, etc. have been defined as instances of Hand, Elbow, etc. not as classes. This was a design decision to improve the performance of the reasoning because the inference mechanism to classify instances supported by the FuzzyDL engine is more efficient than that used for classes.

Once the instances have been created, the relations among them were specified, as illustrated in Table 5, according to the physical connections among the body parts. In addition, the probability of each relation is also specified. Similarly to Table 4, this probability may be used to describe which impairments a patient may have.

Table 5. Ontology developed - Relations between instances

\begin{tabular}{l|lll} 
Instance A & Instance B & Relation & Probability of relation \\
\hline upper_limb & left_arm & hasArm & 1 \\
upper_limb & right_arm & hasArm & 1 \\
right_arm & right_shoulder & hasShoulder & 1 \\
right_arm & right_elbow & hasElbow & 1 \\
right_arm & right_hand & hasHand & 1 \\
left_arm & left_shoulder & hasShoulder & 1 \\
left_arm & left_elbow & hasElbow & 1 \\
left_arm & left_hand & hasHand & 1
\end{tabular}

\subsection{Fuzzy-DL for supporting the rehabilitation of the EIderly}

A fuzzy system, as indicated in (Ross, 2004), is characterized by having logical statements that are not only true or false but can have different degrees of certainty (between 0 and 1). These can be assigned by using different membership functions such as: Trapezoidal function, Triangular function, Left-shoulder function, Crisp function, Linear function and Right shoulder function (see some examples in Table 6).

Fuzzy data can be manipulated, according to (Bobillo \& Straccia, 2016), using fuzzy logic operations such as: conjunction, disjunction, negation and implication. These operators do not 
behave in the same way as in a classic logic, even several authors have given different interpretations to them, such as Łukasiewicz (Giles, 1976), Gödel (Bobillo, Delgado, \& GómezRomero, 2012) y Zadeh (Zadeh, 1965). It was decided to use Łukasiewicz in our proposal because it is the most widely used for inference over ontologies as it deals with unknown degrees of truth while carrying out fuzzy assertions.

In telerehabilitation systems, the use of fuzzy systems emerges naturally. It must be pointed out that much of the information obtained in telerehabilitation is of fuzzy nature, so that it can be defined over fuzzy domains. Our proposal is to use a fuzzy system to determine the degree of mobility of a patient by reasoning over the fuzzy information about the mobility of a body part or area when the reduced proposal of Fugl-Meyer tests (Hsieh et al., 2007) is being executed. However, traditional fuzzy systems do not work with ontologies and cannot benefit from the additional reasoning over classes and relationships that traditional ontological systems do provide. In addition, as discussed in (Bobillo, 2008), classical ontologies are not prepared to represent inaccuracy, vagueness or uncertainty in knowledge preventing classical semantic engines based on OWL from using uncertain information to operate. To overcome these problems hybrid systems that combine both semantic representation mechanisms and fuzzy reasoning strategies have been proposed (Bobillo, 2008) (Lukasiewicz \& Straccia, 2008). In our context the work of (Bobillo, 2008) is particularly interesting because it proposes an extension of the well-known and computable description logic to the fuzzy domain. As indicated in (Bobillo, 2008) this extension brings new interesting affordances with respect to the existing fuzzy-DLs that exhibit different limitations such as:

1. The lack of fuzzy-DL reasoners able to support fuzzy extensions of the languages OWL and OWL 2.

2. The lack of a general formalism able to manage jointly the imprecise and uncertain knowledge in DLs.

Hybrid systems use OWL 2 for the specification of ontologies. Under this standard, there is a need to increase the expressiveness with which imprecise and vague knowledge is represented, so that knowledge affected by uncertainty can be formally defined. According to (Bobillo \& Straccia, 2016), using the Fuzzy-DL tool, an ontology specified by OWL 2 can be used with the extension of fuzzy data to enable fuzzy inferences to take place.

The ontology proposed in Section 3.2 is not compatible with fuzzy data. For this reason, the Fuzzy-DL reasoner presented in (Bobillo \& Straccia, 2016), offers an extension for Protégé that facilitates the integration of fuzzy data (Bobillo, 2015). With these fuzzy elements it is possible to define additional diffuse knowledge that enhances the ontology proposed in 3.2. 
In our context we have defined several fuzzification functions that are used to represent uncertainty over the information that is captured during a rehabilitation test. As an example, for the shoulder flexion test "Flexion of the shoulder from 90 to 180 degrees", a fuzzy type "90To180Flexion" has been defined. This function assigns a value of 0 if the flexion value is less than 90 degrees (see Table 6). As the angle increases, this degree of certainty will be increased until it reaches 1 , when the angle is greater than or equal to 180 degrees. The function that best describes this behaviour corresponds to the "right shoulder" function, as seen in Table 6 ("90To180Flexion"), having q1 equal to 90 degrees and q2 equal to 180 degrees. Other examples of fuzzy data have been presented in Table 6 .

Table 6. Developed ontology - Fuzzy data types

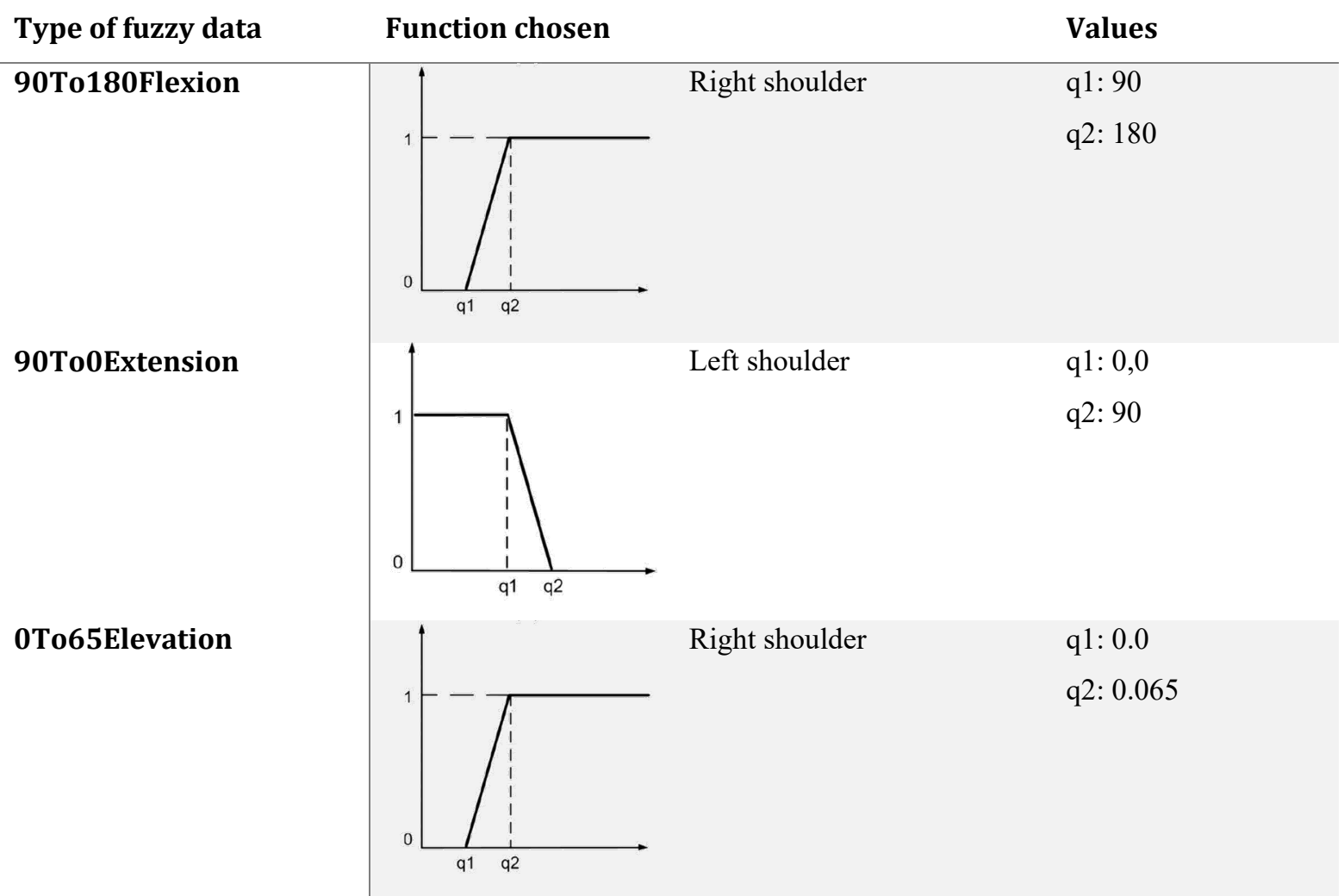

Once the types of fuzzy data have been defined, the fuzzy ontological concepts that allow classifying the instances according to a fuzzy semantics must be defined. Following the example, provided that is needed to specify that a body part may has a flexion between 90 and 180 degrees, the fuzzy concept "High90To180FlexionBodyPart" is defined in a generic way, so that it may be used to describe the flexion of any part of the body. This fuzzy concept is defined in terms of the diffuse data type "90To180Flexion", which takes as domain the values of the ontological property "hasFlexion". It has to be pointed out that "hasFlexion" was defined as an ontological relationship between "Joint" entity as domain and the *real* set as range (see 
Table 3). As can be observed, this facilitates that generic fuzzy concepts can be related to ontological body parts movements, and so that they can be applicable to multiple instances. The fuzzy concepts defined for the fuzzification functions, depicted in Table 6, are presented in Table 7.

Table 7. Ontology developed - Entities related to diffuse concepts

\begin{tabular}{l|ll} 
Fuzzy concept & Type of fuzzy data & Relation \\
\hline High90To180FlexionBodyPart & 90To180Flexion & hasFlexion \\
High90To0ExtensionBodyPart & 90To0Extension & hasExtension \\
High0To65ElevationBodyPart & 0To65Elevation & hasAxisY
\end{tabular}

With these elements being defined, we have a hybrid fuzzy-semantic system that may infer new information about the rehabilitation status of a patient. This can be done because the fuzzy reasoning engine can classify the information according to the fuzzy semantic model using the patient's performance while the rehabilitation tests are being carried out. A simple example of the operation of the hybrid fuzzy-semantic system is shown in Code 1. Assuming a patient has performed a shoulder flexion of 165 degrees. The reasoner is asked about the level of certainty of this flexion being an instance of the diffuse concept High90To180FlexionBodyPart. After applying the inference process, the system computes that a flexion of 165 degrees belongs to the diffuse concept "High90To180FlexionBodyPart" with a certainty value of 0.833 .

Code 1. Example of Fuzzy rule for exercise flexion of right should from 90 to 180

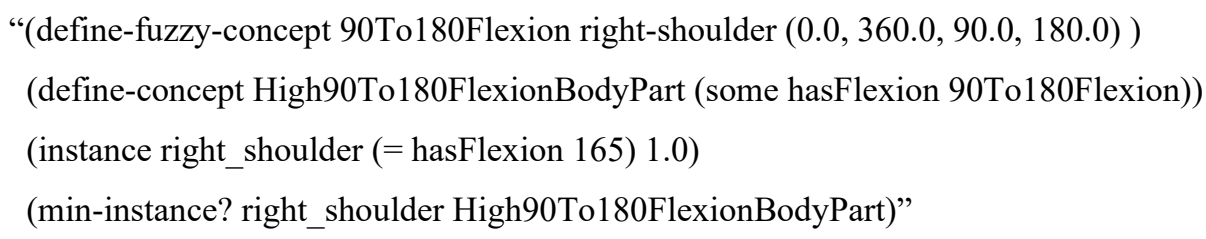

\section{FINE: A Fuzzy ImpairmeNt Evaluation tool}

All the previous fuzzy models have been integrated in our tool FINE (Fuzzy ImpairmeNt Evaluation tool), which consists of two applications: one for the specialist and another for the patient. The general idea pursued by the system is to provide the patient and the expert with a Fugl-Meyer (see section 3.1) assessment of the patient's physical condition based on uncertain information.

The tests described in (Velozo \& Woodbury, 2011) are often used by specialists when evaluating physical problems despite they are very tedious when it comes to evaluating them and their accuracy may be low. For example, one of the tests consists of a shoulder flexion of 
90 to 180 degrees, which in Fugl-Meyer terms would be evaluated as 0,1 or 2. Two patients achieving a flexion of 135 and 150 degrees respectively, would be assigned the same score, even though the second patient is in a better physical condition with respect to this task. In addition, in traditional rehabilitation processes, this task requires the therapist to measure the achieved angle manually, so that he(she) has to be onsite when the task is being carried out to evaluate the progression with respect to previous sessions.

The objective of FINE is to reduce this tedious and complex work. As a first approximation, we have implemented the tests described in (Hsieh et al., 2007) corresponding to the upper extremity. FINE is designed in such a way that the patient does not need to be co-located with the therapist when carrying out the tests. The specialist can assign the tests to patients that may be performed at home, as long as the patients have the technology required by FINE. In order to make this possible, the tests have been designed in such a way that they are simple and interactive for the patient.

It is worth noting that FINE entails an improvement with respect to the Fugl-Meyer evaluations. It is not scored as indicated in (Velozo \& Woodbury, 2011) as a discrete threevalued 0,1 or 2 . Instead, FINE allows the result to be calculated in a continuous interval [0, 1], improving the accuracy of the evaluation. The result of a test is evaluated using the fuzzysemantic hybrid system, explained in detail in Section 3.3. The ontology has fuzzy elements for the inference process supported by the Fuzzy-DL tool (Bobillo \& Straccia, 2016). In the following Sections the Expert and Patient applications are presented.

\subsection{Expert application}

The expert application is used by therapists to assign the tests to their patients, as well as to visualize their results. The application provides therapists with facilities to assign, eliminate or find patients. Moreover, an expert is able to configure the rehabilitation tests to be performed and the number of repetitions of each test for a given session (Figure 2). This information is later provided to the patient so that he(she) is aware of the tests assigned to him(her) by the expert. The system also keeps track of the status of the test so that the therapist knows whether they have been actually carried out by the patient and have access to the evaluation scores (Figure 3). 


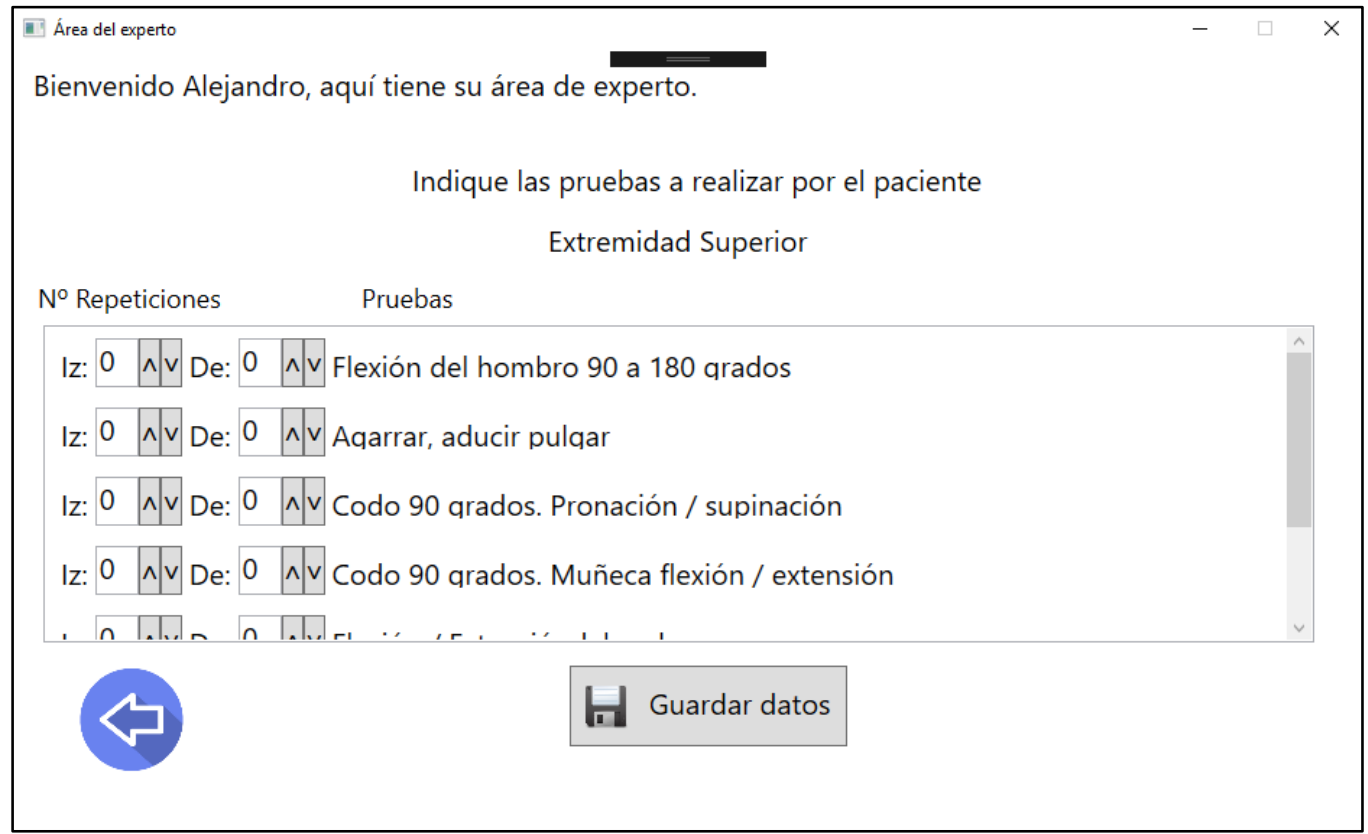

Figure 2. Expert's area, select tests - Expert's application (spanish)

It is important to note that the therapist may visualize several tests simultaneously to carry out a comparative analysis. Using this analysis tool, the therapists may also indicate which period of time and units of time (days, months or years) they are interested in for the visualization. This gives the expert enough flexibility to visualize in a graphical way either fineor coarse-grained data about any number of rehabilitation tests (Figure 3).

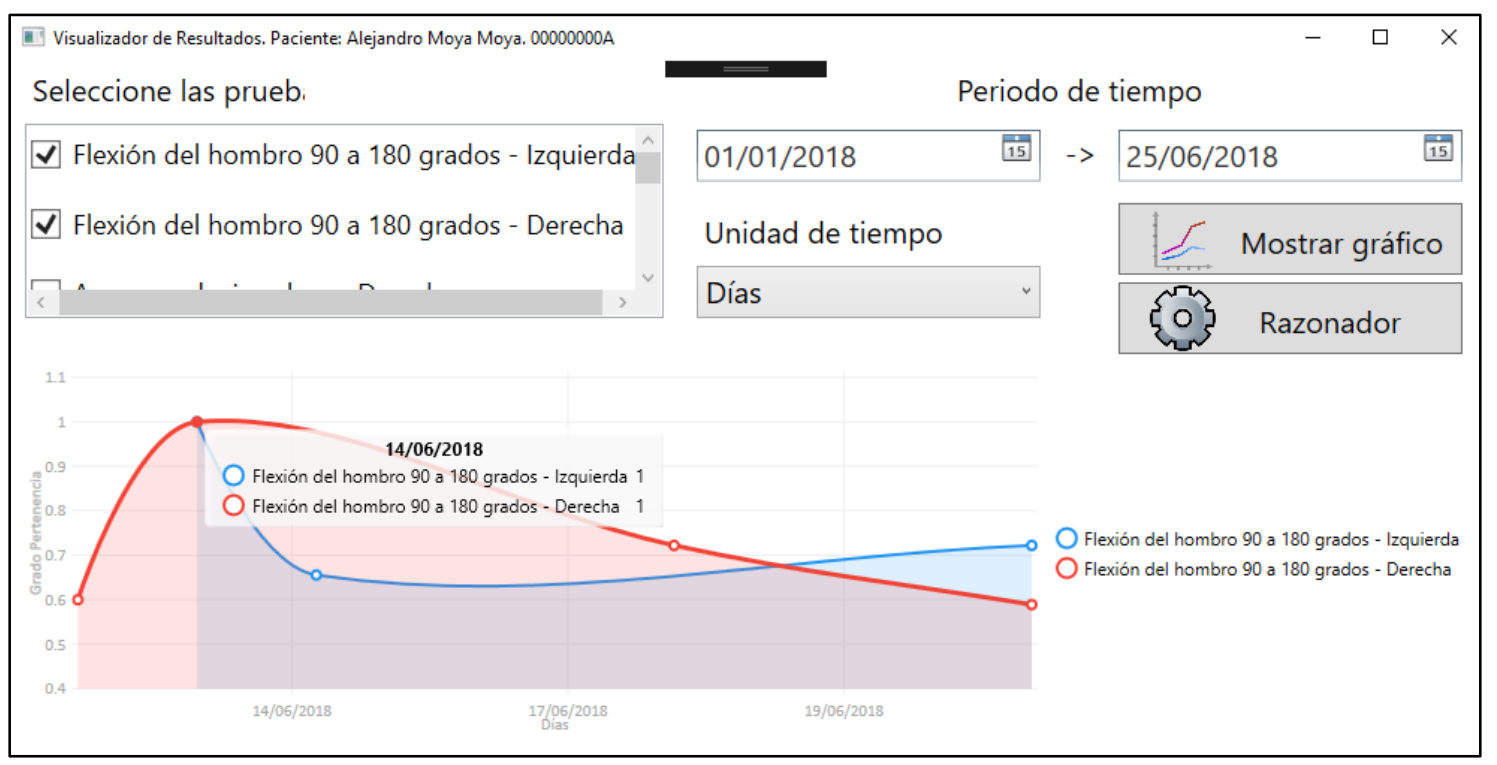

Figure 3. Visualize results, comparison of some results - Expert's application 


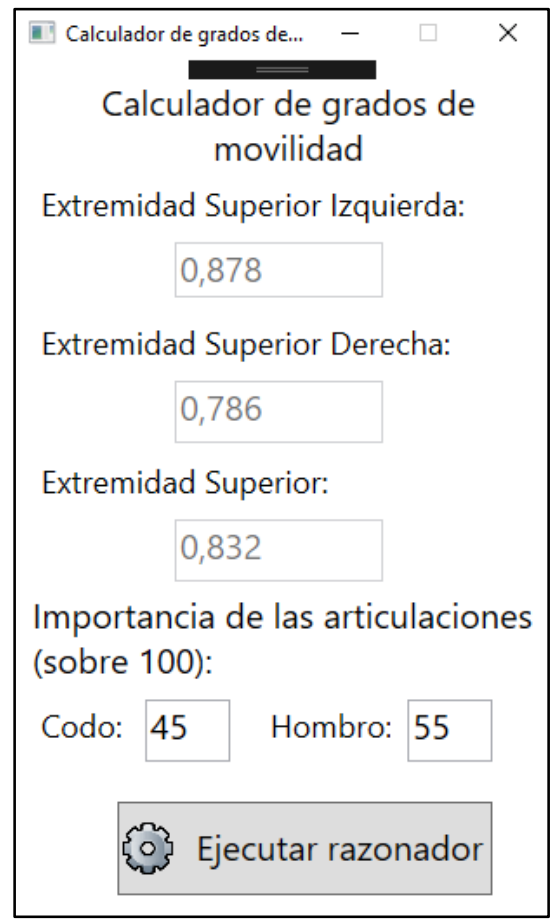

Figure 4. Mobility degrees calculator - Expert's application

Finally, experts may obtain additional information from the fuzzy inference system which performs a data fusion process taking as input the individual mobility scores associated to joints of the patient. In this way, composed concepts related to the mobility degree of anatomical regions may be defined in an ontological way by means of OWL. These regions are then assigned scores by applying fuzzy inference. For example, in Figure 4 the expert obtains fuzzy scores about the mobility degree of the upper (0,832 in Figure 4), right (0,786 in Figure 4)and left limbs $(0,878$ in Figure 4$)$ by applying a fuzzy inference process using the data obtained from the rehabilitation tests of the associated body joints.

In summary, the expert application offers a complete visualization tool of the results of the rehabilitation tests to have a clear view of the patient's recovery progress. Besides, the therapist can assign tests to be performed by patients, and when patients perform them at home, the expert can evaluate them. This system becomes a commodity for patients and therapists, avoiding unnecessary transfers and loss of time in the realization and evaluation of the tests. It also allows a continuous analysis of the patient, thus avoiding idle times between appointments. Another interesting functionality of the system is the ability to reason about the mobility of the patient's limbs according to the relevance expressed as weights that the therapist sets to each individual body joint. The system takes the results of the tests performed by the patient and it is able to interpret these results to offer an overall assessment with respect to a certain limb: upper left extremity, upper right extremity, upper extremity, etc. 


\subsection{Patient application}

This application is the program used by patients to visualize the tests that should perform, as well as to have a real-time interactive control of the execution of these tests.

In this application the patient has access to the pending tests (Figure 5) and has the chance to see a preview of the Kinect sensor camera (showing any detected bones, joints and relative angles) for a better configuration of the device position. It is also shown the local coordinate system of each joint that is used to compute the $3 \mathrm{D}$ joint position.

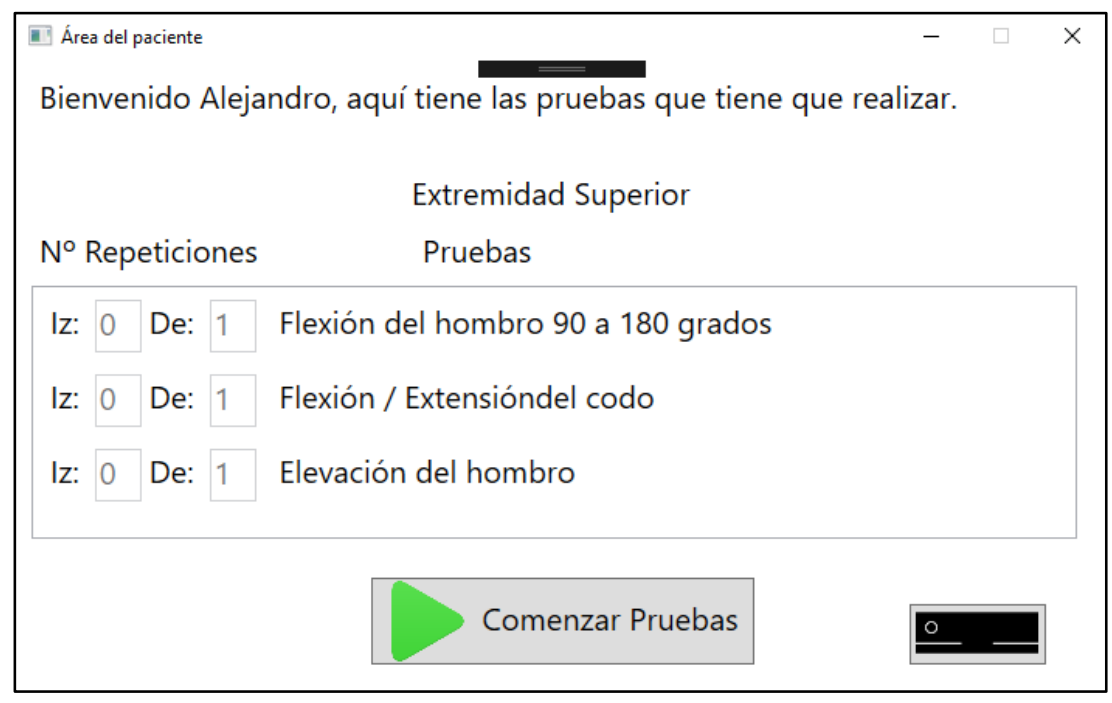

Figure 5. Patient's area, with pending tasks - Patient's application

At any time, the patient may initiate the rehabilitation tests. In this moment the system displays information about the test to be carried out and shows several visual clues for the patient to understand the required exercise. These clues include (Figure 6) a colour video stream of the patient and the environment, a superposed skeleton and a moving blue circle describing the trajectory to be performed with a specific patient's body part (in the example his left arm). During the execution of the tests, the patient is given guidance about the tasks to be performed in several forms (textual, vocal and graphical) and accepts vocal instructions from the user to start, cancel or finalize the test being performed. If the patient decides to cancel the test, then it is marked as unsuccessfully performed. If he(she) decides to finalize it, then the system records the information about its current state even if the test has not been fully performed. It is also important to note that the system indicates the steps to be done in every moment of the test in order to avoid the patient having to read from the screen, just looking at his image would be more than enough. Finally, the system also allows the patient to select whether the colour stream and joints orientations are displayed or not. 
In Figure 6, it can be seen the execution of the test "Shoulder flexion $90^{\circ}$ to $180^{\circ}$ " in which a moving circle is shown to the user who is requested to reach it with an extended arm. The system is monitoring user's movements in order to detect when a test has been completed. It monitors in real time the position of the joints involved in the test. The device provides at all times the position and angles in the 3D space of all the joints and, thus, the system is able to calculate the target point to reach in order to perform a successful test. For example, in test "Shoulder flexion $90^{\circ}$ to $180^{\circ}$ ", the angle made by the shoulder is continuously monitored and compared with the objective to be achieved, in this case $180^{\circ}$. Once the objective is reached, the system finishes the test. If it was the case that the patient could not reach the objective, he(she) would verbally indicate that the test ends with the angle that has been actually reached. In other types of exercises the angle is not measured, but the relative position of the patient with respect to the point to reach. For example, for the test "Shoulder elevation" the position of the shoulder is obtained first and then, it is calculated the relative distance from this point to the target point to be reached.
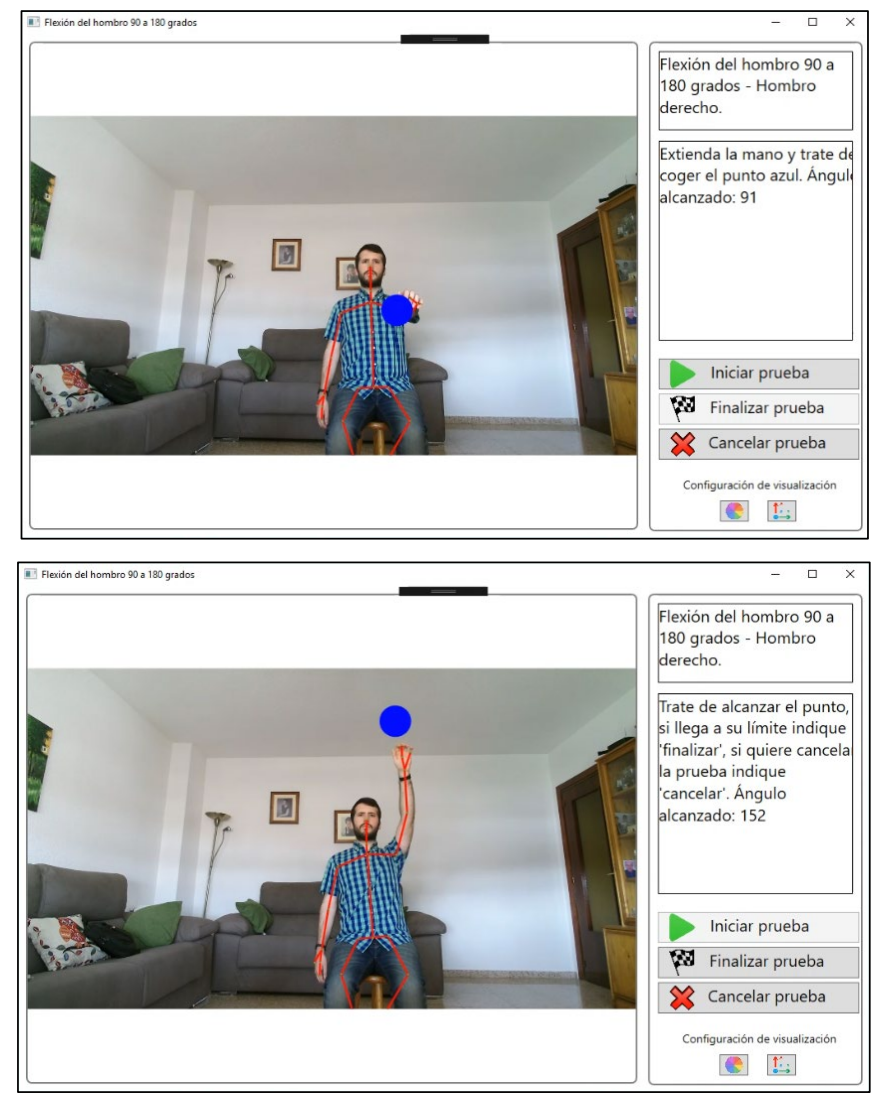

Figure 6. Shoulder flexion $90^{\circ}$ to $180^{\circ}$ - Patient's application

When a test ends, the results about angle, speed, distances, etc., are measured and used as input to generate a fuzzy-semantic hybrid model. The fuzzy-semantic hybrid model described in Section 3.3 cannot be used to perform an inference process, since it needs data for this aim. 
Therefore, the fuzzy-semantic hybrid model is populated with the information captured about user's movements as well as a series of Fuzzy-DL queries related to the information obtained by the Fuzzy-DL inference engine. For instance, in the case of the shoulder flexion test, it includes the flexion made, that is, the angle obtained by the user during the test and the query about belonging to the fuzzy concept "High90To180FlexionBodyPart". Once this has been added to the model, the Fuzzy-DL inference process is executed, and the results stored as part of the test information that will be available for the expert. For example, the patient did a flexion of $165^{\circ}$. The fuzzy-semantic hybrid model described in Section 3.3 is populated with this angle and the query about belonging to the diffuse concept "High90To180FlexionBodyPart" is added. Then FINE includes this information as described in Code 1.

After including these elements, the Fuzzy-DL reasoner is executed and the right_shoulder obtains a degree of belonging to the diffuse concept "High90To180FlexionBodyPart" of 0,8333 . This data is stored and evaluate by the expert.

In summary, the patient application allows him(her) to perform the tests at home in an intuitive way. The user knows at all times the tests he(she) has to perform, the part of the body involved and the number of repetitions. During the execution of a test, the system provides feedback to the user by various means (textual, oral and graphical). In addition, the application allows voice interaction during the performance of the tests. This facility is very important, since the patient cannot use the mouse or keyboard to manipulate the system during a test given the distance to be maintained with respect to the Kinect device.

\subsection{Experimental validation}

The main objective of this evaluation is to perform a preliminary evaluation of the proposed approach in terms of its precision when compared to the traditional method (Fugl-Meyer). It is a validation carried out in an early phase of the tool being the assessment carried out by applying some Fugl-Meyer tests with actual users.

\subsubsection{Participants}

Nine adults whose ages were between 23 and 50 (Mean $(M)=38.556$, Standard Deviation $(\mathrm{SD})=10.967)$ have participated in the tool evaluation process. The gender of the participants is as balanced as possible, with 5 men and 4 women. The participants have been also classified according to their physical constitution, being the groups: ectomorph, endomorph and mesomorph (Carter, 2002), having three participants in each group in order to balance the 
evaluation according to this aspect as well. Table 8 shows the measurements and physical complexions of the participants in the evaluation process.

Table 8. Participants in the evaluation of the proposal

\begin{tabular}{lllllllllll}
\hline ID & Genre & Age & $\begin{array}{l}\text { Physical } \\
\text { complexion }\end{array}$ & $\begin{array}{l}\text { Height } \\
\mathbf{( c m )}\end{array}$ & $\begin{array}{l}\text { Arm } \\
\mathbf{( c m})\end{array}$ & $\begin{array}{l}\text { Shoulder- } \\
\text { Elbow } \\
\mathbf{( c m})\end{array}$ & $\begin{array}{l}\text { Elbow } \\
\mathbf{- H a n d} \\
\mathbf{( c m})\end{array}$ & $\begin{array}{l}\text { Torso } \\
(\mathbf{c m})\end{array}$ & $\begin{array}{l}\text { Shoulder - } \\
\text { Shoulder } \\
\text { (cm) }\end{array}$ & $\begin{array}{l}\text { Hip- } \\
\text { Hip } \\
(\mathbf{c m})\end{array}$ \\
\hline $\mathbf{1}$ & Female & 49 & Endomorph & 166 & 55 & 30 & 25 & 35 & 39 & 40 \\
\hline $\mathbf{2}$ & Male & 50 & Endomorph & 183 & 64 & 35 & 29 & 41 & 41 & 42 \\
\hline $\mathbf{3}$ & Male & 23 & Endomorph & 175 & 56 & 32 & 24 & 44,5 & 41 & 38,5 \\
\hline $\mathbf{4}$ & Female & 25 & Ectomorph & 175 & 66 & 36 & 30 & 45,5 & 39 & 36,5 \\
\hline $\mathbf{5}$ & Female & 44 & Ectomorph & 170 & 55,5 & 28 & 27,5 & 39,5 & 38,5 & 37 \\
\hline $\mathbf{6}$ & Male & 46 & Ectomorph & 191,5 & 70 & 38 & 32 & 53 & 41 & 35 \\
\hline $\mathbf{7}$ & Female & 36 & Mesomorph & 175 & 62 & 34 & 28 & 42 & 40 & 33 \\
\hline $\mathbf{8}$ & Male & 47 & Mesomorph & 182 & 62,5 & 32 & 30,5 & 43 & 43 & 37 \\
\hline $\mathbf{9}$ & Male & 27 & Mesomorph & 185 & 57 & 29 & 28 & 43 & 44 & 36 \\
\hline
\end{tabular}

\subsubsection{Procedure}

For each task, the participant will carry out different tests using the FINE tool. For each test, the participant will repeat the exercise nine times; three times simulating the three possible outcomes of the Fugl-Meyer test 0 (unable to perform), 1 (partial performance) and 2 (close to normal performance). In each repetition the researchers indicated the position to be reached by the participant, so that it corresponds with the Fugl-Meyer rating that is being tested. Given that the tests to be evaluated are angles, it has been decided to divide the range of valid angles in each task into three uniform regions. Finally, we compare the scores obtained with the FINE tool against those that ideally would be obtained by applying the traditional manual method.

\subsubsection{Tasks}

\subsubsection{Shoulder flexion $90^{\circ}$ to $180^{\circ}$}

To carry out this task, the participant has to perform the following steps: (1) The participant has to be located at a distance of two meters from the Kinect device and sitting on a chair or stool; (2) The participant has to extend his arm reaching the point drawn on the computer screen, as shown on top of Figure 6, forming a 90-degree angle with his shoulder and starts the exercise; (3) The participant must try to reach the point so that the angle of flexion of the shoulder increases (bottom of Figure 6). In this task, a researcher conducting the experiment must specify the target position that the participant has to reach to simulate a given Fugl-Meyer score, as indicated in the procedure section. 


\subsubsection{Elbow extension / flexion}

To carry out this second task, the participant has to perform the following steps: (1) The participant has to be located at a distance of two meters from the Kinect device and sitting on a chair or stool; (2) The participant place his elbow being tested close to his body and then extend the arm keeping the elbow position while tries to reach the point visible on the computer screen. In this way, the elbow forms an angle of 90 degrees; (3) The participant in this initial position carries out a flexion of the elbow until he reaches the position indicated by the researcher who is conducting the experiment; (4) After, the participant returns to flex his elbow 90 degrees as indicated in (2); (5) The participant keeping the elbow close to his body, extends his elbow until he reaches the position indicated by the researcher. Again, the flexion and extension target positions simulate conditions that would be scored as 0,1 and 2, respectively, using the FuglMeyer categorization.

\subsubsection{Results}

For each test performed by the participant, the following information was extracted: traditional method score and the score computed by FINE by means of the inference process as a continuous fuzzy value between the closed interval 0 and 1 . The score computed by FINE was discretized to obtain the Fugl-Meyer value. In this way we can compare the discretized value with the score that would manually be assigned if the traditional method was applied.

Table 9. Shoulder flexion (F.) $90^{\circ}$ to $180^{\circ}$ and Fine computed data

\begin{tabular}{|c|c|c|c|c|c|c|c|c|c|c|c|c|c|c|c|c|c|c|}
\hline & \multicolumn{2}{|c|}{$\mathbf{a}$} & \multicolumn{2}{|c|}{ b } & \multicolumn{2}{|c|}{ c } & \multicolumn{2}{|c|}{ d } & \multicolumn{2}{|c|}{$\mathbf{e}$} & \multicolumn{2}{|c|}{ f } & \multicolumn{2}{|c|}{$\sigma$} & \multicolumn{2}{|c|}{ h } & \multicolumn{2}{|c|}{$\mathbf{i}$} \\
\hline ID & F. & Fine & F. & Fine & F. & Fine & F. & Fine & F. & Fine & F. & Fine & F. & Fine & F. & Fine & F. & Fine \\
\hline 1 & 107 & 0 & 114 & 1 & 119 & 1 & 130 & 1 & 144 & 1 & 149 & 1 & 169 & 2 & 173 & 2 & 180 & 2 \\
\hline 2 & 93 & 0 & 110 & 0 & 118 & 1 & 125 & 1 & 130 & 1 & 145 & 1 & 153 & 1 & 163 & 2 & 179 & 2 \\
\hline 3 & 95 & 0 & 115 & 1 & 118 & 1 & 127 & 1 & 132 & 1 & 145 & 1 & 154 & 1 & 164 & 2 & 175 & 2 \\
\hline 4 & 101 & 0 & 117 & 1 & 119 & 1 & 129 & 1 & 133 & 1 & 147 & 1 & 152 & 1 & 168 & 2 & 178 & 2 \\
\hline 5 & 100 & 0 & 109 & 0 & 120 & 1 & 121 & 1 & 135 & 1 & 142 & 1 & 150 & 1 & 172 & 2 & 180 & 2 \\
\hline 6 & 94 & 0 & 100 & 0 & 115 & 1 & 120 & 1 & 139 & 1 & 146 & 1 & 159 & 2 & 175 & 2 & 180 & 2 \\
\hline 7 & 91 & 0 & 115 & 1 & 112 & 0 & 123 & 1 & 140 & 1 & 149 & 1 & 167 & 2 & 179 & 2 & 174 & 2 \\
\hline 8 & 90 & 0 & 119 & 1 & 120 & 1 & 125 & 1 & 148 & 1 & 148 & 1 & 164 & 2 & 164 & 2 & 177 & 2 \\
\hline 9 & 108 & 0 & 118 & 1 & 120 & 1 & 129 & 1 & 148 & 1 & 150 & 1 & 162 & 2 & 175 & 2 & 179 & 2 \\
\hline
\end{tabular}

Table 10. Flexion (F.) /Extension (E.) Elbow and Fine computed data

\begin{tabular}{|c|c|c|c|c|c|c|c|c|c|c|c|c|c|c|c|c|c|c|}
\hline \multirow[b]{2}{*}{ ID } & \multicolumn{2}{|c|}{ a } & \multicolumn{2}{|l|}{ D } & & \multicolumn{2}{|c|}{ d } & & \multicolumn{2}{|l|}{ f } & & \multicolumn{2}{|l|}{ II } & \multicolumn{2}{|l|}{1} \\
\hline & E & ine & F. E. & Fine & E. & Fine & F. E. & Fine & F. E. & Fine & F. E. & Fine & F. E. & Fine & F. E. & Fine & F. E. & Fi \\
\hline 1 & 589 & 0 & $\begin{array}{ll}0 & 102\end{array}$ & 78 & $\begin{array}{ll}0 & 112\end{array}$ & & \begin{tabular}{|ll}
0 & 123
\end{tabular} & 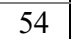 & $\begin{array}{ll}1 & 129\end{array}$ & 15 & 145 & 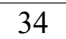 & $\begin{array}{ll}1 & 154\end{array}$ & 23 & $\begin{array}{|ll|}1 & 163 \\
\end{array}$ & 1 & 21 & 0 \\
\hline 2 & 8 & 0 & $\Omega_{0}$ & & 115 & & $\begin{array}{ll}1 & 12\end{array}$ & 51 & 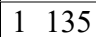 & & 115 & 41 & 1 & & 6 & & . & \\
\hline & 8 & & 10 & & & & 1 & & & & 11 & & 1 & & & & & \\
\hline & 87 & & 1 & 66 & & & 11 & 60 & 1 & & $\begin{array}{ll}1 & 1\end{array}$ & 4 & 1 & & & & & \\
\hline & 290 & & & 81 & 0 & & $\begin{array}{ll}0 & 1\end{array}$ & 52 & & & $\begin{array}{ll}1 & 149\end{array}$ & ( & 158 & 26 & 65 & 3 & & 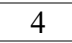 \\
\hline 6 & 38 & 0 & 104 & 76 & 0 & & $\begin{array}{ll}0 & 123\end{array}$ & 53 & 133 & 4 & $\begin{array}{ll}1 & 147\end{array}$ & 37 & $\begin{array}{ll}1 & 151 \\
\end{array}$ & 28 & $\begin{array}{ll}1 & 169\end{array}$ & 1 & 1 & 0 \\
\hline 7 & 784 & 0 & $\begin{array}{ll}0 & 106\end{array}$ & 74 & $\begin{array}{ll}0 & 119\end{array}$ & 63 & $\begin{array}{ll}1 & 124\end{array}$ & 56 & 1136 & 49 & $\begin{array}{ll}1 & 152\end{array}$ & 34 & $\begin{array}{ll}1 & 150\end{array}$ & 19 & $\begin{array}{|ll|}1 & 164\end{array}$ & 14 & 25170 & 1 \\
\hline
\end{tabular}


\begin{tabular}{l|lll|lll|lll|lll|lll|lll|lll|lll|lll}
\hline 8 & 94 & 87 & 0 & 0 & 107 & 78 & 0 & 114 & 65 & 1 & 120 & 55 & 1 & 138 & 51 & 1 & 151 & 36 & 1 & 157 & 27 & 1 & 162 & 9 & 2 & 168 & 0 \\
\hline 9 & 99 & 86 & 0 & 0 & 111 & 77 & 0 & 111 & 68 & 0 & 130 & 57 & 1 & 141 & 46 & 1 & 156 & 39 & 1 & 156 & 23 & 1 & 171 & 8 & 2 & 171 & 6
\end{tabular}

Table 9 and Table 10 show the results of each participant (ID) for the two tests. Each row depicts the degrees reached (F. and E.) and score computed by FINE for the 9 repetitions (a-i) carried out by each participant. We now compare the scores obtained by applying the fuzzy inference with the scores that would be obtained manually by a specialist applying the FuglMeyer criteria (0 - a:c; $1-\mathrm{d}: f ; 2-\mathrm{g}: \mathrm{i})$. Overall, if we take into account the total 162 test repetitions made by all the participants, the algorithm fails to obtain a correct Fugl-Meyer score in 32 cases $(19.753 \%)$, i.e., our proposal is able to categorize properly the performed tests in $80,25 \%$ of the cases. If we consider the results for each type of test, we observe that our fuzzy inference system is able to assign correct scores for the "Shoulder flexion $90^{\circ}$ to $180^{\circ}$ " in $77,77 \%$ of the cases, whereas for the "Flexion / Extension Elbow" test, FINE obtains slightly higher success rates $(82,72 \%)$.

Shoulder flexion 90 으 to 180 은

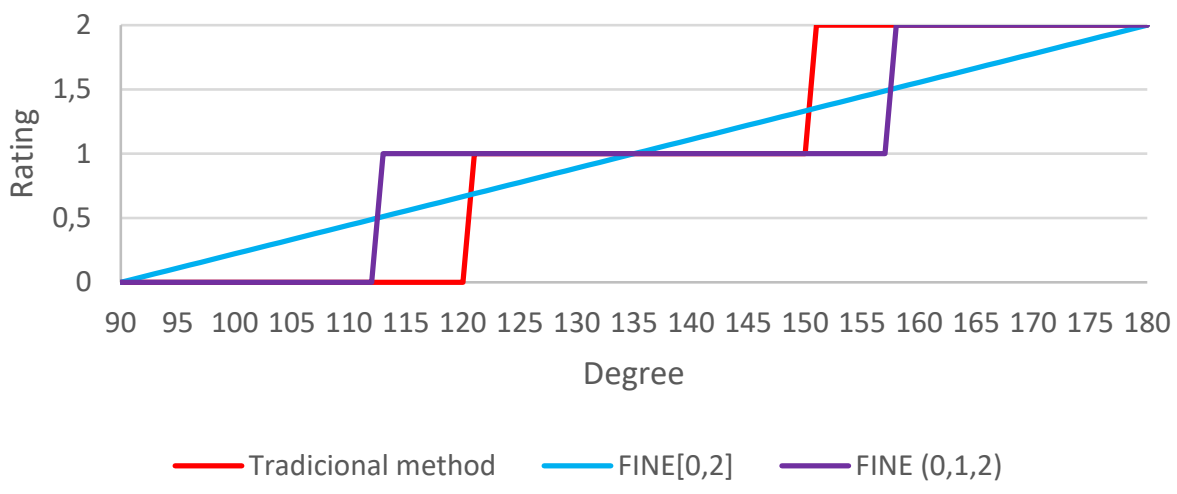

Figure 7. Shoulder flexion $90^{\circ}$ to $180^{\circ}$ - Validation

Extension / Flexion elbow

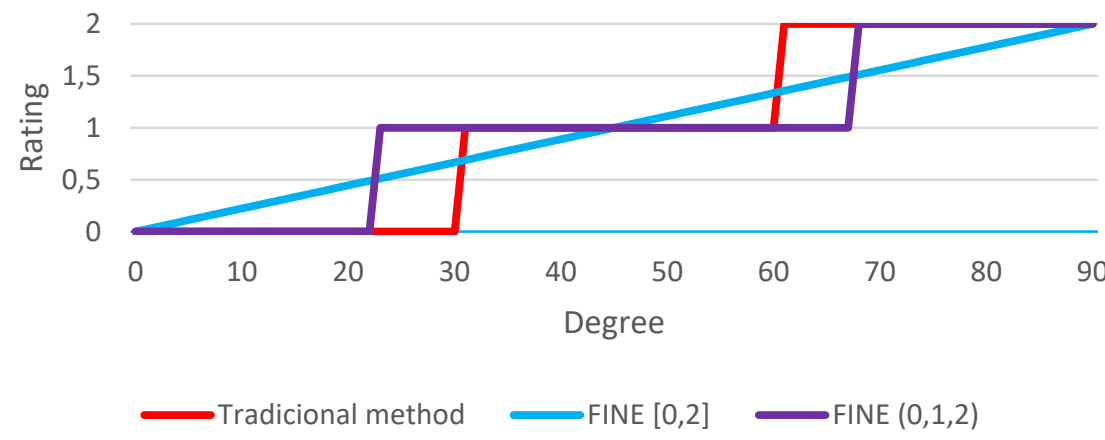

Figure 8. Extension / Flexion elbow - Validation

Figure 7 illustrates how the results obtained in the test "Shoulder flexion $90^{\circ}$ to $180^{\circ}$ " are discretized. We can see how the expert's assessment changes abruptly between 0-1 and 1-2 (red 
line) following a uniform distribution over the complete range of angle values ([90-180]. The blue line shows the score computed by FINE as a continuous value between $0-2$. This score is discretized by our algorithm as shown by the mauve line. It can be observed that both the traditional approach and the discretized proposal behave in a similar way but using thresholds at different angles, as it was decided to split the space into three different uniform areas. However, if we compare the FINE rating [0,2] (blue line) with the traditional method (red line), we can see the benefits of FINE. There is no longer a sharp jump between $0-1$ and 1-2, but the degree reached by the participants is analysed continuously, providing information accordingly. This provides a great amount of information of interest for the experts, since they can evaluate the evolution of the patient throughout his rehabilitation in a more progressive way. Figure 8 shows how the results obtained for the test "Elbow Flexion / Extension" are processed in a similar way. Moreover, we can see a similar phenomenon to that shown in Figure 7, that is, there is an abrupt change between 0-1 and 1-2 when the data are discretized to compare the results with the traditional method.

\section{Conclusions and future work}

It is a fact that the world's population is ageing. According to (Nassabi et al., 2014), the proportion of the older adults $(+65)$ is increasing and is expected to double within the European Union in a period of 50 years. The use of technology to address this problem emerges in a natural way to improve the quality of life of the elderly.

The combination of technology and gerontology is called as Gerotechnology. According to the study realized by (Metz, 2000), among the different domains it addresses, the most important is "Telehealth and telemedicine". This is the reason why this paper is focused on the development of a physical telerehabilitation system.

Many prior works have been proposed to address this problem, such as (Alamri et al., 2010)(Karime et al., 2014)(Chiu et al., 2018), etc. All of them are differentiated, among other things, by the use of an intelligent module for the treatment of the information captured. As we have seen in Section 2, the proposals that make use of fuzzy systems are better than those that do not, because of their ability to deal with the uncertainty. In addition, the inclusion of an intelligent component facilitates the automation of the system, either during the course of the tests or for the subsequent analysis of the data.

Our proposal is aimed at carrying out an analysis of the patient's physical condition. The correct analysis of this physical condition is important, so that the rehabilitation tests are appropriate to the physical condition of the patient. In addition, a continuous analysis of the 
patient's status allows the therapists to adapt the rehabilitation process as needed, without having to wait for the next medical appointment, increasing their effectiveness.

For the correct analysis of the physical state of the patient, this has to be supported by a standard, in our case the Fugl-Meyer standardization. However, this standard has a limitation: the use of only three levels to evaluate the mobility of joints makes it difficult to evaluate the real progress of the patients, as they may get stuck in intermediate scores for a long time. This may lead them to become stale about their rehabilitation. The use of Fuzzy-DL provides a fresh alternative to the automatic evaluation, as the computed result is now continuous instead of discrete.

The use of an ontology that models the human body was also detected as necessary in order to reason about the patient's mobility. The problem with the use of an ontology is that it does not support fuzzy data. This problem has been solved with the use of semantic fuzzy hybrid systems, such as (Bobillo \& Straccia, 2016). In this paper, authors present the Fuzzy-DL tool, a reasoner that facilitates the specification of fuzzy data in an OWL2 ontology and the execution of inference using them. The creation of the fuzzy semantic hybrid system for the analysis of the patient's physical state has led to the creation of the Fuzzy ImpairmeNt Evaluation tool (FINE).

FINE aims to capture the information of the patient (position of joints, angles made, etc) to subsequently make inferences about these data. In this way, the therapist can monitor the scores obtained by the patient for each test and make changes on the assigned rehabilitation process. Another advantage is that the patients can perform their rehabilitation at home without having to move to the medical centre and in a dynamic way, thus allowing flexibility when performing their tasks.

Despite the facilities provided by FINE, it also has some disadvantages. One of them is that both the ontology as well as the fuzzy rules have been defined and implemented in our tool FINE. This facilitates that specialists may use the system without perceiving the complexity behind its implementation. However, in case experts would need to carry out any modification of the fuzzy system, they would need an ontology-developer for this aim as we have not implemented yet any user-friendly tool that facilitates this task.

Several challenges constitute our future work. The main proposal would be the extension of the tests implemented, in order to consider all those described in (Hsieh et al., 2007). As aforementioned, they identified that only those tests illustrated in Table 1 are enough to evaluate the physical state of the patients. In this work, we have focused just on the tests used for the upper limb as a first approach to the standard. In addition, six other tests are included for the 
lower limb. Therefore, it is part of our future work to implement those test for the lower limb, so that all the tests described in Fugl-Meyer could be developed (Velozo \& Woodbury, 2011). It is worth noting that FINE makes possible to perform them with the minimum effort and with the greatest precision.

Another proposal would be to use open source API for the collection devices. FINE has been developed using the Kinect API, a closed source API and only available for Kinect devices. Therefore, after the change of camera, we could make use of an open source API, OpenNI (OpenNI Pioneering Members, 2018), which would allow us to offer the same functionality, to a greater or lesser extent, than Kinect and allows us to use any type of camera. An example of a device that could be used is Orbbec Astra Pro (Orbbec 3D, 2018), a 3D camera that has a functionality very similar to Kinect.

In order to ensure that all tests can be performed and that the data collected are correct, it would be interesting to incorporate new devices in the evaluation of the tests. For example, Leap Motion (Leap Motion INC, 2018) could be incorporated for the detection of the hands, in order to detect them with greater precision and guarantee that the tests related to these extremities will be more successful. Kinect is imprecise at small distances. Therefore, the incorporation of cameras that capture the whole environment would be very interesting, since we would have a three-dimensional model of the user and we would obtain a greater precision than that using just one camera.

In Fugl-Meyer, other patterns to be evaluated during the course of a test are indicated. In (Ar, Jaasko, Leyman, Olsson, \& The, 2010) it is specified that it would be very interesting to measure the pain of the patient during the course of a test, especially in the final part of a test. With Kinect one could make a reading of the facial expressions made by the patient and take into account those expressions that show discomfort, in order to adapt the next tests and store this information in the system.

Another interesting task to carry out in the future is the FINE study with experts. FINE has been developed considering the bibliography on physical rehabilitation. Given the collaboration currently held by the LoUISE group with the National Hospital of Paraplegics, it is estimated that in the coming months a FINE deployment can be carried out in such hospital, in order to carry out an evaluation with both therapists and patients.

Acknowledgements. This research was funded by the Spanish Ministry of Economy and Competitiveness and by EU FEDER funds under project grants TIN2016-79100-R and 
TIN2015-72931-EXP. It has also been funded by the Junta de Comunidades de Castilla-La

Mancha scholarship 2018-UCLM1-9131.

\section{References}

Alamri, A., Cha, J., \& El Saddik, A. (2010). AR-REHAB: An Augmented Reality Framework for Poststroke-Patient Rehabilitation. IEEE Transactions on Instrumentation and Measurement, 59(10), 2554-2563. https://doi.org/10.1109/TIM.2010.2057750

Antoniou, G., \& Van Harmelen, F. (2004). Web ontology language: Owl. In Handbook on ontologies (pp. 67-92). Springer.

Ar, F.-M., Jaasko, L., Leyman, I., Olsson, S., \& The, S. S. (2010). Fugl-Meyer Assessment Lower Extremity (FMA-LE) Assessment of sensorimotor function, (max 2), 1-3.

Bobillo, F. (2008). Managing Vagueness in Ontologies. Universidad de Granada.

Bobillo, F. (2015). The fuzzyDL System. Retrieved July 10, 2018, from http://www.umbertostraccia.it/cs/software/fuzzyDL/fuzzyDL.html

Bobillo, F., Delgado, M., \& Gómez-Romero, J. (2012). DeLorean: A reasoner for fuzzy OWL 2. Expert Systems with Applications, 39(1), 258-272. https://doi.org/10.1016/j.eswa.2011.07.016

Bobillo, F., \& Straccia, U. (2016). The fuzzy ontology reasoner fuzzyDL. Knowledge-Based Systems, 95, 12-34. https://doi.org/10.1016/j.knosys.2015.11.017

Boucenna, S., Narzisi, A., Tilmont, E., Muratori, F., Pioggia, G., Cohen, D., \& Chetouani, M. (2014). Interactive Technologies for Autistic Children: A Review. Cognitive Computation, 6(4), 722-740. https://doi.org/10.1007/s12559-014-9276-X

Bronswijk, J. E. M. H. Van, Bouma, H., \& Fozard, J. L. (2002). Technology for quality of life: an enriched taxonomy. Gerontechnology, 2(2). https://doi.org/10.4017/gt.2002.02.02.001.00

Carter, J. E. L. (2002). The Heath-Carter Anthropometric Somatotype - Instruction Manual. San Diego: State University.

Chiu, Y. H., Chen, T. W., Chen, Y. J., Su, C. I., Hwang, K. S., \& Ho, W. H. (2018). Fuzzy logic-based mobile computing system for hand rehabilitation after neurological injury. Technology and Health Care : Official Journal of the European Society for Engineering and Medicine, 26(1), 17-27. https://doi.org/10.3233/THC-171403

Commission, E. (2006). ICT for Health and i2010 Transforming the European healthcare landscape Towards a strategy for ICT for Health. Network. https://doi.org/10.1017/S1816383112000409

Fernández-Caballero, A., González, P., \& Navarro, E. (2017). Gerontechnologies - Current achievements and future trends. Expert Systems, 34(2), 1-2. https://doi.org/10.1111/exsy.12203

Giles, R. (1976). Łukasiewicz logic and fuzzy set theory. International Journal of ManMachine Studies, 8(3), 313-327. https://doi.org/10.1016/S0020-7373(76)80003-X

Hsieh, Y.-W., Hsueh, I.-P., Chou, Y.-T., Sheu, C.-F., Hsieh, C.-L., \& Kwakkel, G. (2007). Development and Validation of a Short Form of the Fugl-Meyer Motor Scale in Patients With Stroker Stroke, 38(11), 3052-3054. https://doi.org/10.1161/STROKEAHA.107.490730

Karime, A., Eid, M., Alja'Am, J. M., Saddik, A. El, \& Gueaieb, W. (2014). A fuzzy-based adaptive rehabilitation framework for home-based wrist training. IEEE Transactions on Instrumentation and Measurement, 63(1), 135-144. https://doi.org/10.1109/TIM.2013.2277536

Krynicki, K., Jaen, J., \& Navarro, E. (2016). An ACO-based personalized learning technique 
in support of people with acquired brain injury. Applied Soft Computing, 47, 316-331. https://doi.org/10.1016/j.asoc.2016.04.039

Leap Motion INC. (2018). Leap Motion. Retrieved July 10, 2018, from https://www.leapmotion.com/

Lukasiewicz, T., \& Straccia, U. (2008). Managing uncertainty and vagueness in description logics for the Semantic Web. Journal of Web Semantics, 6(4), 291-308. https://doi.org/10.1016/j.websem.2008.04.001

Metz, D. H. (2000). Mobility of older people and their quality of life. Transport Policy, 7(2), 149-152. https://doi.org/10.1016/S0967-070X(00)00004-4

Nassabi, M. H., Akker, H. Den, \& Vollenbroek-, M. (2014). An ontology - based recommender system to promote physical activity for pre - frail elderly, 181-184.

Navarro, E., González, P., López-Jaquero, V., Montero, F., Molina, J. P., \& Romero-Ayuso, D. (2018). Adaptive, Multisensorial, Physiological and Social: The Next Generation of Telerehabilitation Systems. Frontiers in Neuroinformatics, 12(July), 1-7. https://doi.org/10.3389/fninf.2018.00043

OpenNI Pioneering Members. (2018). OpenNI. Retrieved July 10, 2018, from http://openni.ru/about/index.html

Orbbec 3D. (2018). Orbbec Astra Pro. Retrieved July 10, 2018, from https://orbbec3d.com/product-astra-pro/

Rodríguez, A. C., Roda, C., Montero, F., González, P., \& Navarro, E. (2016). An Interactive Fuzzy Inference System for Teletherapy of Older People. Cognitive Computation, 8(2), 318-335. https://doi.org/10.1007/s12559-015-9356-6

Ross, T. J. (2004). Fuzzy Logic With Engineering. Second Edition.

Shaughnessy, M., Resnick, B. M., \& Macko, R. F. (2006). Testing a Model of Post-Stroke Exercise Behavior. Rehabilitation Nursing, 31(1), 15-21. https://doi.org/10.1002/j.20487940.2006.tb00005.x

Su, C. J., Chiang, C. Y., \& Huang, J. Y. (2014). Kinect-enabled home-based rehabilitation system using Dynamic Time Warping and fuzzy logic. Applied Soft Computing Journal, 22(November 2010), 652-666. https://doi.org/10.1016/j.asoc.2014.04.020

Velozo, C. A., \& Woodbury, M. L. (2011). Translating measurement findings into rehabilitation practice: An example using Fugl-Meyer Assessment-Upper Extremity with patients following stroke. The Journal of Rehabilitation Research and Development, 48(10), 1211. https://doi.org/10.1682/JRRD.2010.10.0203

W3C. (2012). OWL 2 Web Ontology Language. Retrieved July 10, 2018, from https://www.w3.org/TR/owl2-overview/

Zadeh, L. A. (1965). Fuzzy sets. Information and Control, 8(3), 338-353. https://doi.org/10.1016/S0019-9958(65)90241-X

Zhang, Z., Fang, Q., \& Gu, X. (2014). Fuzzy inference system based automatic Brunnstrom stage classification for upper-extremity rehabilitation. Expert Systems with Applications, 41(4 PART 2), 1973-1980. https://doi.org/10.1016/j.eswa.2013.08.094

Zhao, W., Reinthal, M. A., Espy, D. D., \& Luo, X. (2017). Rule-Based Human Motion Tracking for Rehabilitation Exercises: Realtime Assessment, Feedback, and Guidance. IEEE Access, 5, 21382-21394. https://doi.org/10.1109/ACCESS.2017.2759801 